TRANSACTIONS OF THE AMERICAN MATHEMATICAL SOCIETY

Volume 348, Number 10, October 1996

\title{
ON THE HOMOLOGY SPECTRAL SEQUENCE FOR TOPOLOGICAL HOCHSCHILD HOMOLOGY
}

\author{
THOMAS J. HUNTER
}

\begin{abstract}
Marcel Bökstedt has computed the homotopy type of the topological Hochschild homology of $\mathbb{Z} / p$ using his definition of topological Hochschild homology for a functor with smash product. Here we show that easy conceptual proofs of his main technical result of are possible in the context of the homotopy theory of $S$-algebras as introduced by Elmendorf, Kriz, Mandell and May. We give algebraic arguments based on naturality properties of the topological Hochschild homology spectral sequence. In the process we demonstrate the utility of the unstable "lower" notation for the Dyer-Lashof algebra.
\end{abstract}

\section{INTRODUCTION}

The aim of this paper is to provide a first tool for calculating the homology of the topological Hochschild homology, $\operatorname{thh}(R)$, of a commutative $S$-algebra spectrum $R$. The interest in this calculation lies in the close relation between topological Hochschild homology and algebraic $K$-theory. Throughout this paper, unless we mention otherwise, $p$ is an odd prime.

Our main theorem states that in the spectral sequence calculating the $\bmod p$ homology of thh $(R)$ the first canonically possible differential is a certain primary operation. Here, $H \mathbb{Z} / p$ is a cell $S$-algebra (in the sense of [5, VII]) representing reduced homology modulo $p$, and $R$ represents any $q$-cofibrant $S$-algebra. We will now state this theorem and give a brief proof. The rest of the paper will be devoted to providing the necessary definitions and proofs of statements made during this proof.

The properties of the Hochschild homology spectral sequence which we shall use are enumerated in Theorem 8. In Section 7 we state how these properties are contained in [5].

Theorem 1. Let $R$ be a q-cofibrant $S$-algebra. Suppose $x \in(H \mathbb{Z} / p)_{n} R$ with $n$ odd and positive. Then in the spectral sequence

$$
\mathrm{HH}_{*}\left((H \mathbb{Z} / p)_{*} R\right) \Rightarrow(H \mathbb{Z} / p)_{*} \operatorname{thh}(R)
$$

$\gamma_{p^{k}}[x]$ lives to $E^{p-1}$ and

$$
d^{p-1}\left(\gamma_{p^{k}}[x]\right)=\left[c \beta Q^{\frac{n+1}{2}} x\right] \gamma_{p^{k}-p}[x]
$$

for some nonzero $c \in \mathbb{Z} / p$.

Received by the editors October 14, 1994

1991 Mathematics Subject Classification. Primary 55S12, $19 D 55$.

(C)1996 American Mathematical Society 
Here $\mathrm{HH}_{*}$ denotes Hochschild homology, the square brackets are standard bar construction notation, $\gamma_{p^{k}}[x]$ denotes the $p^{k}$-th divided power of $[x]$, the $Q^{i}$ are Steinberger's Dyer-Lashof operations, and the spectral sequence referred to is given by [5, IX.2.8]. Marcel Bökstedt obtained this result for $R$ a commutative functor with smash products in [1].

Remark 2. In Section 6 we show that the theorem also holds for odd negative $n$ given one further assumption about the Hochschild homology spectral sequence.

Remark 3. In particular $H \mathbb{Z} / p$ is a $q$-cofibrant $S$-algebra and given any $S$-algebra $A$ there is a weak equivalence $\Lambda A \rightarrow A$ of $S$-algebras with $\Lambda A q$-cofibrant. See [5, VII] for details.

Once one accepts the properties of the category of spectra our proof of this more general result is also technically simpler. In fact, the proof consists of observing the necessary naturality, identifying a universal example, and making the calculation for the universal example.

Proof. As constructed in [5], $H \mathbb{Z} / p \wedge_{S} R$ is weakly equivalent to the usual smash product. Hence, we may represent $x$ by a map $S^{n} \rightarrow(H \mathbb{Z} / p) \wedge_{S} R .(H \mathbb{Z} / p) \wedge_{S}(-)$ is also the left adjoint to the forgetful functor from $H \mathbb{Z} / p$ algebras to $S$-algebras, so we can extend this to a map of $H \mathbb{Z} / p$-algebras (that is to say $S$-algebras under $H \mathbb{Z} / p)$

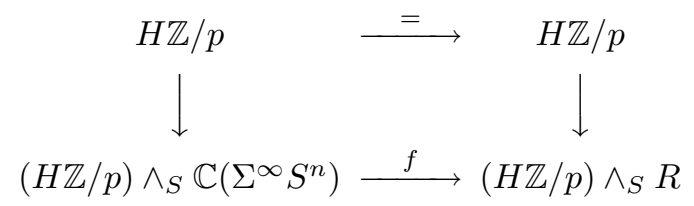

by adjointness. Here $\mathbb{C}$ is the left adjoint constructed in [5, II.4.1]. By the results of Section $2,(H \mathbb{Z} / p)_{*} f$ is a map of algebras over the Dyer-Lashof algebra which takes $\iota_{n}$ to $x$ and by the properties listed in Section $3 f$ induces a map of spectral sequences. In Sections 4 and 5 the formula for $d^{p-1}$ is proved for $(H \mathbb{Z} / p) \wedge S$ $\mathbb{C} \Sigma^{\infty}\left(S^{n}\right)$. The theorem follows.

Acknowledgment. I would like to thank Jim McClure for introducing thh to me, Frank Peterson for introducing the Adem relations to me, and everyone involved with this project for their patience.

\section{Naturality of the Dyer-Lashof operations}

We now remark that the Dyer-Lashof operations are natural transformations with the appropriate domain. Dyer-Lashof operations are defined in the homology of $H_{\infty}$-spectra in [2]. Since $S$-algebras are $E_{\infty}$ spectra by [5, II.4.6] and $E_{\infty}$ spectra are automatically $H_{\infty}$ spectra by neglect of structure, these operations are also defined in the homology of $S$-algebras. The following result is a restatement of part of McClure's Lemma [2, IX.1.3] as it applies to his definition (in [2, IX.1]) of Dyer-Lashof operations in the homology of $H_{\infty}$ ring spectra. Let $\mathcal{C}$ be the full subcategory of $H_{\infty}$ spectra under $H \mathbb{Z} / p$ generated by objects of the form $(H \mathbb{Z} / p) \wedge X$ for $X$ an $H_{\infty}$ spectrum.

Lemma 4 (McClure). The Dyer-Lashof operations are natural operations $\pi_{i} \rightarrow \pi_{j}$ of functors from $\mathcal{C}$ to the category of Abelian groups. 
We note here that with almost no extra effort, the restriction to a full subcategory in McClure's definition may be dropped yielding a definition which is both simpler and more general. In the following lemma and discussion, we freely use the notation of [2, IX.1].

Lemma 5. Fix a given $H_{\infty}$ spectrum, E. Given any $e \in E_{m}\left(D_{p} S^{n}\right)$, there is a natural transformation $Q_{e}: \pi_{n} \rightarrow \pi_{m}$ of functors from the category of $H_{\infty}$ spectra under $E$ to the category of Abelian groups. The restriction of these operations to the full sub-category $\mathcal{C}$ of objects of the form $E \wedge X$ gives McClure's operations.

Proof. If $e$ is represented by $g: S^{m} \rightarrow E \wedge D_{p} S^{n}$ and $x \in \pi_{n} A$ is represented by $f: S^{n} \rightarrow A$, we define $Q_{e} x$ to be represented by the following composite.

$$
S^{m} \stackrel{g}{\longrightarrow} E \wedge D_{p} S^{n} \stackrel{E \wedge D_{p} f}{\longrightarrow} E \wedge D_{p} A \stackrel{E \wedge \xi}{\longrightarrow} E \wedge A \stackrel{\mu}{\longrightarrow} A
$$

Naturality is immediate, and agreement with McClure's definition when $A=E \wedge X$ follows from the commutativity of the following diagram which is a consequence of the definitions in $[2, \mathrm{I}]$.

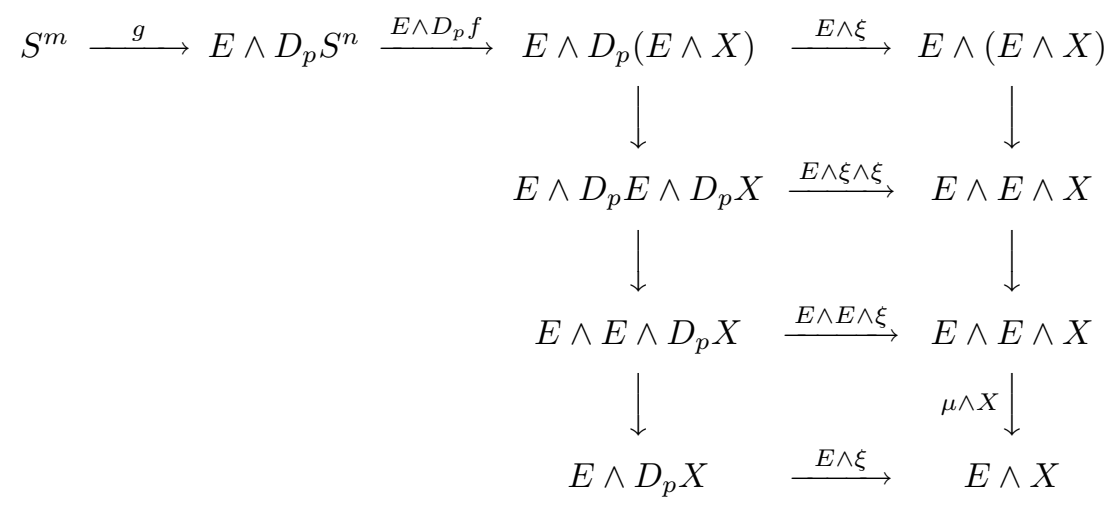

Here the composite of the upper row with the right hand column is our definition and the composite of the left hand and bottom maps give McClure's.

Remark 6 . Since any $H \mathbb{Z} / p$-algebra is an $H_{\infty}$ spectrum under $H \mathbb{Z} / p$ by neglect of structure, the Dyer-Lashof operations are also natural transformations of the functors $\pi_{*}$ from $H \mathbb{Z} / p$-algebras to Abelian groups.

\section{HoCHSCHILD HOMOLOGY}

In this section we recall the definition of Hochschild homology and state those properties of topological Hochschild homology which we shall use in our arguments.

Suppose that $\mathcal{C}$ is a symmetric monoidal category with product $\wedge: \mathcal{C} \times \mathcal{C} \rightarrow \mathcal{C}$ and unit $U \in \operatorname{obj} \mathcal{C}$. Write $A^{k}$ for the $k$-fold product $A \wedge \cdots \wedge A$ and $A^{0}$ for $U$. We will often abuse notation by omitting mention of the natural isomorphisms making $\wedge$ associative and unital.

Definition 7. Let $A$ be a monoid in $(\mathcal{C}, \wedge)$ with structure maps $\mu: A \wedge A \rightarrow A$ and $\epsilon: U \rightarrow A$. Let $M$ be a left and right (unital) $A$ module with structure maps $\mu_{L}: A \wedge M \rightarrow M$ and $\mu_{R}: M \wedge A \rightarrow M$. We define the cyclic bar construction CB. $(A ; M)$ to be the simplicial object with $k$-simplices $\mathrm{CB}_{k}(A ; M)=M \wedge A^{k}$, face maps $d_{i}: \mathrm{CB}_{k}(A ; M) \rightarrow \mathrm{CB}_{k-1}(A ; M)$, and degeneracy maps $s_{i}: \mathrm{CB}_{k}(A ; M) \rightarrow$ 
$\mathrm{CB}_{k+1}(A ; M)$ defined by the following equations in which we have repeatedly suppressed mention of the identity maps for $A$ and $M$.

$$
\begin{aligned}
d_{0} & =\mu_{R}:(M \wedge A) \wedge A^{k-1} \rightarrow M \wedge A^{k-1}, \\
d_{i} & =\mu: M \wedge A^{i-1} \wedge(A \wedge A) \wedge A^{k-i-1} \rightarrow M \wedge A^{k-1} \quad(0<i<k), \\
d_{k} & =T \circ \mu_{L} \circ T: M \wedge A^{k-1} \wedge A \stackrel{\cong}{\longrightarrow} A^{k-1} \wedge(A \wedge M) \rightarrow A^{k-1} \wedge M \stackrel{\cong}{\longrightarrow} M \wedge A^{k-1}, \\
s_{i} & =\epsilon: M \wedge A^{i} \wedge A^{k-i} \rightarrow M \wedge A^{i} \wedge A \wedge A^{k-i} \quad(0 \leq i \leq k) .
\end{aligned}
$$

When $\mathcal{C}$ is the category of graded modules over a ring $k, \mathrm{CB}_{\bullet}$ is a simplicial graded vector space and we recover the usual Hochschild homology $\mathrm{HH}_{*}(A ; M)=$ $\pi_{*}(\mathrm{CB} \cdot(A ; M))$. When $M=A$ we omit it in this and similar notation, so, for example $\mathrm{HH}_{*}(A)=\mathrm{HH}_{*}(A ; A)$. When $\mathcal{C}$ is the category of $S$-modules (or $H \mathbb{Z} / p$ modules) $\mathrm{CB} .(A ; M)$ is the simplicial $S$-module (or $H \mathbb{Z} / p$-module) underlying the definition of thh (or thh ${ }^{H \mathbb{Z} / p}$ ) given in [5, IX.2.1].

We now list the properties of thh which we will use to obtain our results. The proofs all are in or follow fairly quickly from [5] and are given in Section 7. Write $\rightarrow \rightarrow$ for the category with two objects and one non-identity map between them and write $\mathcal{A}^{\mathcal{B}}$ for the category of functors from $\mathcal{B}$ to $\mathcal{A}$. Write $\mathcal{E}$ for the category of $S$-algebras constructed in [5] and $\mathcal{E}_{H \mathbb{Z} / p}$ for the comma category of maps $H \mathbb{Z} / p \rightarrow B$. In other words, $\mathcal{E}_{H \mathbb{Z} / p}$ is the category of $H \mathbb{Z} / p$-algebras. Recall that for any space $X$ equivalent to a CW-complex we have an equivalence $C_{+}\left(\Sigma^{\infty} X\right) \simeq \Sigma^{\infty}\left(Q X_{+}\right)$. Whenever $Y$ is a based space, we will write $H \mathbb{Z} / p_{*} Y$ as an abbreviation for $H \mathbb{Z} / p_{*} \Sigma^{\infty} Y$.

Theorem 8 (Elmendorf, Kriz, Mandell and May). For each object $H \mathbb{Z} / p \rightarrow A \rightarrow$ $B$ in $\mathcal{E}_{H \mathbb{Z} / p}^{\cdot \rightarrow}$, there is a spectrum $\operatorname{thh}^{H \mathbb{Z} / p}(A ; B)$ and a spectral sequence converging to $\pi_{*} \operatorname{thh}^{H \mathbb{Z} / p}(A ; B)$ with the following properties. We will call the spectral sequence the mod $p$ Hochschild homology spectral sequence and we will call $\operatorname{thh}^{H \mathbb{Z} / p}(A ; B)$ the mod $p$ topological Hochschild homology of A with coefficients in B.

(1) $\operatorname{thh}^{H \mathbb{Z} / p}$ and the mod $p$ Hochschild homology spectral sequence are functors with domain the category $\mathcal{E}_{H \mathbb{Z} / p}^{\cdot}$.

(2) When $A \rightarrow B$ is a map in $\mathcal{E}$ and $A$ and $B$ are $q$-cofibrant $H \mathbb{Z} / p$-algebras, the mod $p$ Hochschild homology spectral sequence for $H \mathbb{Z} / p \wedge_{S} A \rightarrow H \mathbb{Z} / p \wedge_{S} B$ has $E_{* *}^{2}=\mathrm{HH}_{*}\left(H \mathbb{Z} / p_{*} A ; H \mathbb{Z} / p_{*} B\right)$.

(3) If

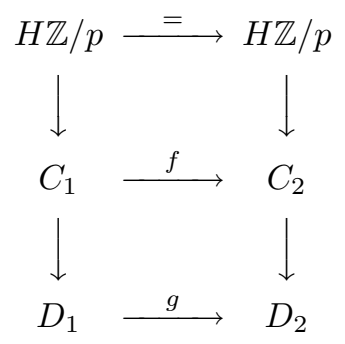

is a map in $\mathcal{E}_{H \mathbb{Z} / p}$ with $f$ and $g$ weak equivalences and with $C_{1}, D_{1}, C_{2}$ and $D_{2}$ all $q$-cofibrant $H \mathbb{Z} / p$-algebras, then the map induced on $E^{2}$ terms in the mod $p$ Hochschild homology spectral sequence is an isomorphism and therefore $\operatorname{thh}^{H \mathbb{Z} / p}(f ; g)$ is also a weak equivalence. 
(4) The mod $p$ Hochschild homology spectral sequence is a spectral sequence of algebras.

(5) Write $E_{* *}^{r}$ for the mod $p$ Hochschild homology spectral sequence for the identity map on $H \mathbb{Z} / p \wedge_{S} \mathbb{C} \Sigma^{\infty} S^{n}$ so we have

$$
\begin{aligned}
E_{* *}^{2}=\mathrm{HH}_{*}\left(H \mathbb{Z} / p_{*} \mathbb{C} \Sigma^{\infty} S^{n} ; H \mathbb{Z} / p_{*} \mathbb{C} \Sigma^{\infty} S^{n}\right) \Rightarrow \\
\pi_{*} \operatorname{thh}^{H \mathbb{Z} / p}\left(H \mathbb{Z} / p \wedge_{S} \mathbb{C} \Sigma^{\infty} S^{n} ; H \mathbb{Z} / p \wedge_{S} \mathbb{C} \Sigma^{\infty} S^{n}\right) .
\end{aligned}
$$

Let ${ }^{\prime} E^{r}{ }_{* *}$ be the usual bar construction spectral sequence with

$$
{ }^{\prime} E_{* *}^{2}=\operatorname{Tor}^{H \mathbb{Z} / p_{*} Q S_{+}^{n}}(\mathbb{Z} / p, \mathbb{Z} / p) \Rightarrow H \mathbb{Z} / p_{*} Q S_{+}^{n+1} .
$$

Then there is an isomorphism of spectral sequences

$$
E_{* *}^{r} \stackrel{\cong}{\longrightarrow} H \mathbb{Z} / p_{*} Q S_{+}^{n} \otimes_{\mathbb{Z} / p}{ }^{\prime} E_{* *}^{r} .
$$

\section{A convenient formula for $H \mathbb{Z} / p_{*} C X$}

A formula for $H \mathbb{Z} / p_{*} C X$ where $X$ is a unital spectrum and $C$ is Steinberger's construction [7] is given in IX.2.1 of [2]. This formula provides the necessary input to our spectral sequences because [5] shows that $C\left(X \vee S^{0}\right)$ is weakly equivalent to $\mathbb{C} X$. In this section we recall this formulae and introduce some notation making it easier to manipulate.

We will use "lower index" notation for the Dyer-Lashof algebra in order to deal conveniently with excess and admissibility conditions. Up to a multiplicative constant this is a reindexing of the same lower notation as in [2] and in the unstable case in [4] and [9]. Rather than stabilizing the unstable formulae from the earlier references we will base all our definitions and formulae on [2].

Definition 9. Let $p$ be an odd prime and $x \in H \mathbb{Z} / p_{*} E$ with $E$ an $E_{\infty}$ spectrum. For any $j \in Z$ define

$$
Q_{j} x= \begin{cases}Q^{(|x|+j) / 2} x & \text { when }(|x|+j) / 2 \text { is an integer, } \\ Q_{j} x=0 & \text { otherwise. }\end{cases}
$$

The following observations are immediate from this definition and the properties listed in Theorem [2, III.1.1.7]. $\left|Q_{j} x\right|=p|x|+(p-1) j \equiv|x|(\bmod 2)$ so $Q_{j} Q_{k}=0$ unless $j \equiv k(\bmod 2)$ and $Q_{j} \beta Q_{k}=0$ unless $j \equiv k+1(\bmod 2)$. Furthermore, $Q_{j} Q_{k} x$ is admissible for $x$ of any degree if and only if $j \leq k$ and $Q_{j} \beta Q_{k} x$ is admissible if and only if $j \leq k-1 . Q_{0} x=x^{p}$ and $Q_{j} x=0$ if $j<0$. (Note, of course, that the $Q_{j}$ may lower degree.)

When $p$ is odd statements (3) and (4) of Theorem [2, III.1.1.7] imply the following statement about the instability of the action of the Dyer-Lashof algebra on the homology of $H_{\infty}$ spectra.

$$
Q^{I} x=0 \text { if } 2\left(p i_{1}-\epsilon_{1}\right)-\left|Q^{I}\right|<|x|
$$

where $Q^{I}=\beta^{\epsilon_{1}} Q^{i_{1}} \beta^{\epsilon_{2}} Q^{i_{2}} \ldots \beta^{\epsilon_{n}} Q^{i_{n}}$. Letting $Q^{I}=\beta^{\epsilon_{1}} Q^{i_{1}} Q^{I^{\prime}}$ we may rewrite this as the unstable condition: $Q^{i_{1}} Q^{I^{\prime}} x=0$ if $2 i_{1}<\left|Q^{I^{\prime}} x\right|$ and $\beta Q^{i_{1}} Q^{I^{\prime}} x=0$ if $Q^{i_{1}} Q^{I^{\prime}} x=\left(Q^{I^{\prime}} x\right)^{p}$. Hence if $Q_{I} x=Q_{i_{1}} \beta^{\epsilon_{2}} Q_{i_{2}} \ldots \beta^{\epsilon_{n}} Q_{i_{n}} x$ then $Q_{I} x$ may be nonzero only if $i_{1} \geq 0$ and $\beta Q_{I} x$ may be nonzero only if $i_{1}>0$. (The reader may easily check that the same is true when $p=2$ if we define $Q_{j} x=Q^{|x|+j} x$. Similarly, simpler versions of all our results involving the Dyer-Lashof algebra hold 
when $p=2$ but we will not mention them further since our application is for odd p.)

We are now ready to restate Theorem IX.2.1 of [2]. Let $X$ be a unital spectrum in which the unit $\eta: \Sigma^{\infty} S^{0} \rightarrow X$ is an essential map of spectra. Let $v$ be a generator of the image of $H \mathbb{Z} / p_{*} \eta$. Choose $A \subset H \mathbb{Z} / p_{*} X$ so that $A \cup\{v\}$ is a basis for $H \mathbb{Z} / p_{*} X$. Let $C A$ be the free commutative algebra generated by the set $\left\{Q_{I} x \mid x \in A\right\}$ where the sequence $I=\left(\epsilon_{1}, i_{1}, \ldots \epsilon_{n}, i_{n}\right)$ is subject to the following conditions:

$$
\begin{gathered}
n \geq 0, \\
0<i_{1} \leq i_{2} \leq \cdots \leq i_{n}, \\
\epsilon_{i} \in\{0,1\}, \\
i_{t} \equiv \begin{cases}i_{t+1} \quad(\bmod 2) & \text { if } \epsilon_{t+1}=0 \\
i_{t+1}+1 \quad(\bmod 2) & \text { if } \epsilon_{t+1}=1,\end{cases} \\
i_{n} \equiv|x| \quad(\bmod 2) .
\end{gathered}
$$

Unless explicitly stated otherwise, when we write $Q_{I} x$ we will assume that the above conditions hold. Write $\epsilon I=\sum_{i=1}^{n} \epsilon_{i}$ and $\alpha I=i_{1}$ unless $n=0$ in which case $\alpha I=\infty$. As in [2] the $E_{\infty}$ structure of $C X$ and the standard inclusion $X \rightarrow C X$ give a map $\lambda: C A \rightarrow H \mathbb{Z} / p_{*} C X$. The following theorem is Theorem IX.2.1 of [2].

Theorem 10 (McClure). $\lambda$ is an isomorphism.

$$
\text { 5. A COUnting ARgument For } \Sigma^{\infty} Q S_{+}^{n} \text { FOR } n>0
$$

As noted in Assumptions 8, the mod $p$ Hochschild homology spectral sequence for $\Sigma^{\infty} Q S_{+}^{n} \simeq \mathbb{C} \Sigma^{\infty} S^{n}$ is just the tensor product of the constant spectral sequence $H \mathbb{Z} / p_{*} Q S_{+}^{n}$ with the usual bar construction spectral sequence

$$
\operatorname{Tor}^{H \mathbb{Z} / p_{*} Q S_{+}^{n}}(\mathbb{Z} / p, \mathbb{Z} / p) \Rightarrow H \mathbb{Z} / p_{*} Q S_{+}^{n+1} .
$$

In this section we give a simple counting argument to find the "first" differential in this spectral sequence. All our other results will follow from this one by naturality arguments.

Proposition 11. Let $n$ be a positive odd integer and let $\iota \in H \mathbb{Z} / p_{*} Q S_{+}^{n}$ be the fundamental class. In the bar construction spectral sequence

$$
\operatorname{Tor}^{H \mathbb{Z} / p_{*} Q S_{+}^{n}}(\mathbb{Z} / p, \mathbb{Z} / p) \Rightarrow H \mathbb{Z} / p_{*} Q S_{+}^{n+1}
$$

the element $[\iota|\ldots| \iota]=\gamma_{p}[\iota]$ lives to $E^{p-1}$ and $d^{p-1} \gamma_{p}[\iota]=c\left[\beta Q_{1} \iota\right]$ for some nonzero constant $c$. Furthermore $E^{2}=E^{p-1}, E^{p}=E^{\infty}$ and $d^{p-1}$ is given on the algebra generators of $E^{p-1}$ by the formula $d^{p-1} \gamma_{p^{k}}\left[Q_{I} \iota\right]=c\left[\beta Q_{1} Q_{I} \iota\right] \gamma_{p^{k}-p}\left[Q_{I} \iota\right]$ for any odd dimensional $Q_{I} \iota$ satisfying the conditions (1).

Remark 12. A chain level argument using [6] giving this result and showing that $c$ can be taken to be 1 is indicated on page 25 of [4]. Here we show that a much simpler argument may be given in the special case we are interested in and obtain the more general formula (and its extension to $T H H$ of spectra) by naturality arguments.

Proof. We will use the facts that this spectral sequence is a spectral sequence of Hopf algebras over the Dyer-Lashof algebra and that we know both the $E^{2}$ term and the object to which the spectral sequence converges. 
Write $P(S)$ for the polynomial algebra generated by the set $S, E(S)$ for the exterior algebra generated by $S, \Gamma(S)$ for the divided polynomial algebra generated by $S$, and $T(S)$ for the polynomial algebra uniformly truncated at height $p$ generated by $S$. Note that $\left|Q_{I} x\right| \equiv \epsilon I+|x| \equiv \alpha I+\epsilon_{1}(\bmod 2)$.

Using the formula from Section 4 and the fact that $n$ is odd we get the following formulae in which all congruences are modulo 2.

$$
\begin{aligned}
& (H \mathbb{Z} / p)_{*} Q S_{+}^{n}=P\left(Q_{I} \iota_{n} \mid \epsilon I \equiv 1\right) \otimes E\left(Q_{I} \iota_{n} \mid \epsilon I \equiv 0\right), \\
& (H \mathbb{Z} / p)_{*} Q S_{+}^{n+1}=P\left(Q_{I} \iota_{n+1} \mid \epsilon I \equiv 0\right) \otimes E\left(Q_{I} \iota_{n+1} \mid \epsilon I \equiv 1\right), \\
& \operatorname{Tor}^{(H \mathbb{Z} / p)_{*} Q S_{+}^{n}}(\mathbb{Z} / p, \mathbb{Z} / p)=E\left(\left[Q_{I} \iota_{n}\right] \mid \epsilon I \equiv 1\right) \otimes \Gamma\left(\left[Q_{I} \iota_{n}\right] \mid \epsilon I \equiv 0\right) .
\end{aligned}
$$

Here we are continuing to use the assumption that the conditions (1) hold. We will use these formulae to see where the Hilbert series for the $E^{2}$ term and the $E^{\infty}$ term first differ. First, we factor the Hilbert series of each of the terms on the right hand side of equation (2). We factor the exterior term by distinguishing generators by whether or not $\alpha I=1$.

$$
\begin{aligned}
h\left(E\left(\left[Q_{I} \iota_{n}\right] \mid \epsilon I \equiv 1\right)\right)=h\left(E\left(\left[Q_{I} \iota_{n}\right] \mid \epsilon I \equiv 1, \alpha I>1\right)\right) \\
\cdot h\left(E\left(\left[\beta Q_{1} Q_{I} \iota_{n}\right] \mid \epsilon I \equiv 0, \alpha I>0\right)\right)
\end{aligned}
$$

We factor the divided power term by using $h(\Gamma(a))=h(T(a)) h\left(\Gamma\left(\gamma_{p}(a)\right)\right)$ and distinguishing truncated polynomial generators by the number of 1 's in $I$.

$$
\begin{gathered}
h\left(\Gamma\left(\left[Q_{I} \iota_{n}\right] \mid \epsilon I \equiv 0\right)\right)=h\left(T\left(\left[\left(Q_{1}\right)^{i} Q_{I} \iota_{n}\right] \mid \epsilon I \equiv 0, \alpha I>1, i \geq 0\right)\right) \\
\cdot h\left(\Gamma\left(\gamma_{p}\left[Q_{I} \iota_{n}\right] \mid \epsilon I \equiv 0, \alpha I>0\right)\right)
\end{gathered}
$$

From the equality of total degrees $\left|\left[Q_{i} \iota_{n}\right]\right|=\left|Q_{i-1} \iota_{n+1}\right|$ we deduce $\left|\left[Q_{I} \iota_{n}\right]\right|=$ $\left|Q_{I-1} \iota_{n+1}\right|$ when $I=\left(\epsilon_{1}, i_{1}, \ldots, \epsilon_{n}, i_{n}\right)$ and $I-1=\left(\epsilon_{1}, i_{1}-1, \ldots, \epsilon_{n}, i_{n}-1\right)$. Furthermore, if $|y| \equiv|x|+1(\bmod 2)$ then $Q_{I-1} y$ satisfies the conditions (1) if and only if $Q_{I} x$ satisfies (1) and $\alpha I>1$. These observations allow us to identify some of the factors as follows.

$$
\begin{aligned}
h\left(E\left(\left[Q_{I} \iota_{n}\right] \mid \epsilon I \equiv 1, \alpha I>1\right)\right) & =h\left(E\left(Q_{I} \iota_{n+1} \mid \epsilon I \equiv 1\right)\right) \\
h\left(T\left(\left[\left(Q_{1}\right)^{i} Q_{I} \iota_{n}\right] \mid \epsilon I \equiv 0, \alpha I>1, i \geq 0\right)\right) & \\
& =\prod_{i \geq 0} h\left(T\left(\left(Q_{I} \iota_{n+1}\right)^{p^{i}} \mid \epsilon I \equiv 0, \alpha I>0\right)\right) \\
& =h\left(P\left(Q_{I} \iota_{n+1} \mid \epsilon I \equiv 0, \alpha I>0\right)\right)
\end{aligned}
$$

Substituting, we get

$$
\begin{aligned}
& h\left(\operatorname{Tor}^{(H \mathbb{Z} / p)_{*} Q S_{+}^{n}}(\mathbb{Z} / p, \mathbb{Z} / p)\right)=h\left((H \mathbb{Z} / p)_{*} Q S_{+}^{n+1}\right) \\
& \cdot h\left(E\left(\left[\beta Q_{1} Q_{I} \iota_{n}\right] \mid \epsilon I \equiv 0, \alpha I>0\right)\right) \\
& \cdot h\left(\Gamma\left(\gamma_{p}\left[Q_{I} \iota_{n}\right] \mid \epsilon \equiv 0, \alpha I>0\right)\right) \text {. }
\end{aligned}
$$

In particular, the smallest degree in which $h\left(\operatorname{Tor}^{(H \mathbb{Z} / p)_{*} Q S_{+}^{n}}(\mathbb{Z} / p, \mathbb{Z} / p)\right)$ differs from $h\left((H \mathbb{Z} / p)_{*} Q S_{+}^{n+1}\right)$ is $\left|\left[\beta Q_{1} \iota_{n}\right]\right|=p(n+1)-1$. We deduce there are $r_{0}, s_{0}$, and $t_{0}$ such that $s_{0}+t_{0}=p(n+1), d_{s_{0}, t_{0}}^{r_{0}}$ is nonzero, and $d_{s, t}^{r}=0$ for any $(r, s, t)$ with $r>1$ and $s+t<p(n+1)$. Since $E_{*, *}^{*}$ is a homology spectral sequence of algebras, $d_{s_{0}, t_{0}}^{r_{0}}$ annihilates decomposables and $s_{0}>1$. Examination of formula 
(2) shows that the indecomposables of filtration greater than 1 in total degree $p(n+1)$ are a rank one vector space generated by $\gamma_{p}\left[\iota_{n}\right]$. Since there must be a differential in this degree, $\gamma_{p}\left[\iota_{n}\right]$ must support it. Since $E_{*, *}^{*}$ is a spectral sequence of coalgebras, $d_{s_{0}, t_{0}}^{r_{0}} \gamma_{p}\left[\iota_{n}\right]$ must be primitive. $\left[\beta Q_{1} \iota_{n}\right]$ spans the primitives with total degree $p(n+1)-1$ so it must be in the image of $d_{s_{0}, t_{0}}^{r_{0}}$. The formula for $d^{p-1} \gamma_{p}[\iota]$ follows. By naturality the formula for $d^{p-1} \gamma_{p}[x]$ follows for any $x$ with positive odd degree. The rest of the proposition follows as in $[11,6.10]$ because the spectral sequence is a spectral sequence of Hopf algebras.

By the splitting result in Theorem 8 the formula also holds in the mod $p$ Hochschild homology spectral sequence for $\mathbb{C} \Sigma^{\infty} S^{n}$ for positive $n$. This finishes the proofs of the statements used in the proof of Theorem 1 given in Section 1. In fact, we have demonstrated the following more general fact.

Theorem 13. Let $A$ be an object in $\mathcal{E}_{H \mathbb{Z} / p}$. Suppose $x \in \pi_{n} A$ with $n$ odd and positive. Then in the spectral sequence

$$
\mathrm{HH}_{*}\left(\pi_{*} A\right) \Rightarrow \pi_{*} \operatorname{thh}^{H \mathbb{Z} / p}(A)
$$

$\gamma_{p^{k}}[x]$ lives to $E^{p-1}$ and

$$
d^{p-1}\left(\gamma_{p^{k}}[x]\right)=\left[c \beta Q^{\frac{n+1}{2}} x\right] \gamma_{p^{k}-p}[x]
$$

for some nonzero $c \in \mathbb{Z} / p$.

\section{Negative Dimensions}

More care must be taken when $n$ is not positive. In general there is no canonically defined coproduct - even in the universal examples - and there may be no bottom dimensional elements. For example the elements $\left(Q_{-n}\right)^{i} \iota_{n}$ with $i \geq 0$ provide infinitely many elements of degree $n$ in $Q H \mathbb{Z} / p_{*} \mathbb{C} S^{n}$ when $n$ is not positive, so the rank of the module of indecomposables of the same bidegree as $\gamma_{p}\left[\iota_{n}\right]$ is certainly not one. In this section we note that if there is an appropriate internal action of the Dyer-Lashof algebra on the mod $p$ Hochschild homology spectral sequence, we can use the Adem relations and this action of the Dyer-Lashof algebra to avoid these difficulties.

In [8] Ligaard and Madsen provide an internal action of the Dyer-Lashof algebra in the mod 2 homology bar construction spectral sequence for the homology of the de-looping of an infinite loop space. It is reasonable to hope that similar techniques provide a vertical action in the mod $p$ Hochschild homology spectral sequence.

Assumption 14. The columns $E_{s, *}^{r}$ in the $\bmod p$ Hochschild homology spectral sequence are modules over the Dyer-Lashof algebra. The differentials respect this module structure. The action is determined by the Cartan formula on the cyclic bar construction:

$$
Q^{i}\left[x_{1}|\ldots| x_{s}\right]=\sum_{i_{1}+\cdots+i_{s}=i}\left[Q^{i_{1}} x_{1}|\ldots| Q^{i_{s}} x_{s}\right]
$$

We record the result of applying this Cartan formula to the divided power generators in the mod $p$ Hochschild homology spectral sequence in the following corollary. 
Corollary 15. Let $x \in A$ be an odd dimensional element in a graded algebra $A$ on which the Dyer-Lashof algebra acts. Then in $\mathrm{CB}_{*}(A ; A)$ we have

$$
\begin{aligned}
Q^{i} \gamma_{s}[x] & =\sum_{i_{1}+\cdots+i_{s}=i}\left[Q^{i_{1}} x|\ldots| Q^{i_{s}} x\right]=\sum_{\begin{array}{c}
s_{1} j_{j}+\cdots+s_{t} j_{t}=i \\
s_{1}+\cdots+s_{t}=s \\
j_{1}<\cdots<j_{t}
\end{array}} \gamma_{s_{1}}\left[Q^{j_{1}} x\right] \ldots \gamma_{s_{t}}\left[Q^{j_{t}} x\right], \\
\beta \gamma_{s}[x] & =\sum_{0 \leq i<s}(-1)^{i} \overbrace{[x|\ldots| x}^{i \text { terms }}|\beta x| x|\ldots| x]=[\beta x] \gamma_{s-1}[x] .
\end{aligned}
$$

Proof. This is a straightforward application of the Cartan formula.

When using lower index notation in the spectral sequence, we will use total degree to calculate the indices. Thus, for example, $Q_{j}[x]=Q^{\frac{|x|+1+j}{2}}[x]=\left[Q^{\frac{|x|+1+j}{2}} x\right]=$ $\left[Q_{j+1} x\right]$. Despite this shift in dimension inherent in the lower index notation, we will often find it convenient because the formulae for the differentials are simpler in this notation.

The following lemma will enable us to extend our theorem to classes in negative dimensions.

Lemma 16. If $x$ is a nonzero element in $\mathrm{HH}_{*}\left(H \mathbb{Z} / p_{*} \mathbb{C} S^{n}\right)$ with $x \neq 1$ and $N$ is an integer there is $i>N$ with $Q_{i} x \neq 0$.

Note that since $\mathrm{HH}_{*}\left(H \mathbb{Z} / p_{*} \mathbb{C} S^{n}\right)=H \mathbb{Z} / p_{*} \mathbb{C} S^{n} \otimes \operatorname{Tor}_{s, t}^{H \mathbb{Z} / p_{*} \mathbb{C} S^{n}}(\mathbb{Z} / p, \mathbb{Z} / p)$ it suffices to prove the theorem for $x \neq 0,1$ in $\operatorname{Tor}_{s, t}^{H \mathbb{Z} / p_{*}} \mathbb{C} S^{n}(\mathbb{Z} / p, \mathbb{Z} / p)$.

Before we prove this lemma we will show how it implies Theorem 13 when $n$ is negative.

Proposition 17. Let $A$ be an object in $\mathcal{E}_{H \mathbb{Z} / p}$. Suppose $x \in \pi_{n} A$ with $n$ odd. If there are vertical operations as asserted in Assumption 14 in the spectral sequence

$$
\mathrm{HH}_{*}\left(\pi_{*} A\right) \Rightarrow \pi_{*} \operatorname{thh}^{H \mathbb{Z} / p}(A)
$$

then $\gamma_{p^{k}}[x]$ lives to $E^{p-1}$ and

$$
d^{p-1}\left(\gamma_{p^{k}}[x]\right)=\left[c \beta Q^{\frac{n+1}{2}} x\right] \gamma_{p^{k}-p}[x]
$$

for some nonzero $c \in \mathbb{Z} / p$.

Proof. By our naturality arguments, it suffices to prove the theorem for $x=\iota_{n} \in$ $H \mathbb{Z} / p_{n} \mathbb{C} S^{n}$. We will proceed by induction on $k$. First consider the case $k=1$. If $\gamma_{p}\left[\iota_{n}\right]$ did not live to $E^{p-1}$ then for some $j$ with $2 \leq j<p-1$ we would have $d^{j} \gamma_{p}\left[\iota_{n}\right] \neq 0$. By Corollary 15 and Theorem $13 Q^{i} d^{j} \gamma_{p}\left[\iota_{n}\right]=d^{j} \gamma_{p}\left[Q^{i / p} \iota_{n}\right]=0$ whenever $\left|Q^{i / p} \iota_{n}\right|>0$. This contradicts Lemma 16 so $\gamma_{p}\left[\iota_{n}\right]$ does live to $E^{p-1}$. Now let $d^{p-1} \gamma_{p}\left[\iota_{n}\right]-\left[\beta Q_{1} \iota_{n}\right]=u$ and suppose that $u \neq 0$. Applying the Cartan formula we get $Q^{i} d^{p-1} \gamma_{p}\left[\iota_{n}\right]=d^{p-1} \gamma_{p}\left[Q^{i / p} \iota_{n}\right]$. Applying the Adem relations (See below for a convenient form.) we get $Q^{i}\left[\beta Q_{1} \iota_{n}\right]=\left[\beta Q_{1} Q^{i / p} \iota_{n}\right]$. Hence Theorem 13 implies $Q^{i} u=0$ for all large enough $i$, which again contradicts Lemma 16 .

When $k>1$ the proof is essentially the same, but the applications of Corollary 15 are more complicated. If $2 \leq j<p-1$ Corollary 15 and the inductive hypothesis give $Q^{i} d^{j} \gamma_{p^{k}}\left[\iota_{n}\right]=d^{j} \gamma_{p^{k}}\left[Q^{i / p^{k}} \iota_{n}\right]$. Therefore, in order to avoid a contradiction 
between Theorem 13 and Lemma 16, $\gamma_{p^{k}}\left[\iota_{n}\right]$ must live to $E^{p-1}$. Similarly, Corollary 15 and the inductive hypothesis also give

$$
\begin{aligned}
& Q^{i}\left(d^{p-1} \gamma_{p^{k}}\left[\iota_{n}\right]-\left[\beta Q_{1} \iota_{n}\right] \gamma_{p^{k}-p}\left[\iota_{n}\right]\right) \\
& \quad=d^{p-1} \gamma_{p^{k}}\left[Q^{i / p^{k}} \iota_{n}\right]-\left[\beta Q_{1} Q^{i / p^{k}} \iota_{n}\right] \gamma_{p^{k}-p}\left[Q^{i / p^{k}} \iota_{n}\right] .
\end{aligned}
$$

Once again, in order to avoid a contradiction between Theorem 13 and Lemma 16 , we deduce $d^{p-1} \gamma_{p^{k}}\left[\iota_{n}\right]=\left[\beta Q_{1} \iota_{n}\right] \gamma_{p^{k}-p}\left[\iota_{n}\right]$. This determines the formula on the algebra generators, hence the proof is finished.

In order to prove our lemma, we will need to refer to the Adem relations. The Adem relations also take on a simple form in the lower notation. The reindexed Adem relations are given by the following proposition:

Proposition 18. Whenever $l>m$ are nonnegative elements of $\mathbb{Z}$ congruent to the degree of $x$ modulo 2 we have the following relations.

$$
\begin{aligned}
& Q_{l} Q_{m} x=\sum_{\substack{l>t>m \\
t \equiv|x|}}(-1)^{\frac{l-t}{2}}\left(p \frac{t-m}{2}-\frac{l-m}{2}, \frac{l-t}{2}-1\right) Q_{l-p(t-m)} Q_{t} x \\
& Q_{l} \beta Q_{m} x= \\
& \sum_{\substack{l>t>m \\
t \equiv|x| \underset{(\bmod 2)}{ }}}(-1)^{\frac{l-t-1}{2}}\left(p \frac{t-m}{2}-\frac{l-m-1}{2}, \frac{l-t-1}{2}\right) \beta Q_{l-p(t-m)-1} Q_{t} x, \\
& -\sum_{\substack{l>t>m \\
t \equiv|x|}}(-1)^{\frac{l-t-1}{2}}\left(p \frac{t-m}{2}-\frac{l-m-1}{2}-1, \frac{l-t-1}{2}\right) Q_{l-p(t-m)} \beta Q_{t} x .
\end{aligned}
$$

Proof. The reader may verify that these are literal translations of the relations in [2] III.1.1.7 and III.1.1.8. See also [3], in which a similar formula appears which, if $p$ is odd, is applicable only when $x$ is of even degree. It is interesting to note that the degree of $x$ does not appear in our version of these formulae.

Proof of Lemma 16 for elements of homological degree one. In this case we have $x$ $\in Q H \mathbb{Z} / p_{*} \mathbb{C} S^{n}$.

Order the collection of sequences $I$ first by length $l(I)$ and then lexicographically from the left ignoring the exponents of $\beta$. (Except for the possibility of a leading $\beta$, if $Q_{I}$ is nonzero, the exponents of $\beta$ can be deduced from the parities of the $i_{t}$.) This gives a linear ordering of the standard basis for the elements of any degree in $Q H \mathbb{Z} / p_{*} \mathbb{C} S^{n}$. In any $\mathbb{Z} / p$ vector space with an ordered basis we will say that $y$ has leading term $y_{0}$ if $x=c y_{0}+y$ with $c$ nonzero in $\mathbb{Z} / p, y_{0}$ a basis element, and $y$ a linear combination of basis elements smaller than $y_{0}$. We will abuse this language by declaring the leading term of 0 to be less than any basis element.

Notice that when $I$ has $i_{s}>i_{s+1}$ for some $s$ applying the Adem relation for $Q_{i_{s}} Q_{i_{s+1}}$ or $Q_{i_{s}} \beta Q_{i_{s+1}}$ replaces $Q_{I}$ with a linear combination of terms of the form $Q_{J}$ with $J$ less than $I$.

For each admissible sequence $I=\left(\epsilon_{1}, i_{1}=\alpha I, \ldots, \epsilon_{t}, i_{t}=\omega I\right)$ we will define an equivalence class $f(I)\left(\bmod 2 p^{l I}\right)$ such that the following statements hold for any $x \in Q H \mathbb{Z} / p_{*} \mathbb{C} S^{n}$ with leading term $Q_{I} \iota_{n}$. If $i>p^{l I-1} \omega I$ is an integer with $i \equiv f(I)$ $\left(\bmod 2 p^{l I}\right)$ then $Q_{i} x$ has leading term $Q_{I} Q_{i^{\prime}} \iota_{n}$ for some $i^{\prime}$. ( $i^{\prime}$ is the unique integer making $\left|Q_{i} x\right|=\left|Q_{I} Q_{i^{\prime}} \iota\right|$.) If $i \leq p^{l I-1} \omega I$ and $i \equiv f(I)$ then $Q_{i} x$ has leading term 
less than or equal to $Q_{I} Q_{i^{\prime}} \iota$. If $i \not \equiv f(I), Q_{i} x$ has leading term less than $Q_{I} Q_{j} \iota$ for every $j$. The lemma will follow immediately for the case $s=1$.

Define $f(I)$ by induction on the length of $I$ as follows: If $l I=0$, then $x=$ $Q_{I} \iota_{n}=\iota_{n}$. In this case, let $f(I)=n$. The claimed properties are trivial. If $l I>0$, let $f(I)$ be the unique equivalence class such that $f(I) \equiv \alpha I+\epsilon_{1}(\bmod 2 p)$ and $\frac{f(I)-\alpha I-\epsilon_{1}}{p}+\alpha I \equiv f\left(\epsilon_{2}, i_{2}, \ldots\right)\left(\bmod 2 p^{l I-1}\right)$. Inspection of the Adem relations shows that for any integer $f$ such that $f \equiv \alpha I+\epsilon_{1}(\bmod 2 p)$ and $f \geq \alpha I$ the leading term in the expansion $Q_{f} \beta^{\epsilon_{1}} Q_{\alpha I}$ is $\beta^{\epsilon_{1}} Q_{\alpha I} Q_{\frac{f-\alpha I-\epsilon_{1}}{p}+\alpha I}$ and for any integer $f$ such that $f \not \equiv \alpha I+\epsilon_{1}(\bmod 2 p)$ or $f<\alpha I$ the leading term in the expansion $Q_{f} \beta^{\epsilon} Q_{\alpha I}$ is less than $\beta^{\epsilon_{1}} Q_{\alpha I} Q_{\left[\frac{f-\alpha I-\epsilon_{1}}{p}\right]+\alpha I}$. Therefore the inductive hypothesis implies that $f(I)$ has the stated property.

Proof of Lemma 16 for elements of homological degree $s>1$. Let $C_{*}$ be the chain complex underlying the cyclic bar construction, so $C_{n}=\mathrm{CB}_{n}\left(H \mathbb{Z} / p_{*} \mathbb{C} S^{n} ; \mathbb{Z} / p\right)$, the differential is the alternating sum of the face maps and

$$
H\left(C_{*}, d\right)=\operatorname{Tor}^{H \mathbb{Z} / p_{*}} \mathbb{C} S^{n}(\mathbb{Z} / p, \mathbb{Z} / p) .
$$

Let

$$
W_{*}=E\left(\left[Q_{I} \iota_{n}\right]|| Q_{I} \iota_{n} \mid \text { is even }\right) \otimes \Gamma\left(\left[Q_{I} \iota_{n}\right]|| Q_{I} \iota_{n} \mid \text { is odd }\right) \subset C_{*} .
$$

Let $Z_{*} \subset W_{*}$ be defined by the same formula as $W_{*}$ but with the added restriction that $\alpha I>0$ for each $I$ which appears. We have already implicitly used the fact that $W_{*}$ is a submodule of cycles in $C_{*}$ whose inclusion yields an isomorphism upon passing to homology. $W_{*}$ is visibly a sub-algebra of $C_{*}$. That it is also closed under the action of the Dyer-Lashof algebra is a consequence of the formulae in Corollary 15. Every term $\left[Q_{I_{1}} \iota_{n}|\ldots| Q_{I_{s}} \iota_{n}\right]$ of any boundary $y \in W_{s}$ has $\alpha I_{j}=0$ for some $j$. Call such a term a term with leading zeros. Now order the standard basis for $C_{s}=$ $\left(H \mathbb{Z} / p_{*} \mathbb{C} S^{n}\right)^{\otimes s}$ lexicographically from the left. If $x$ is a nonzero element of $C_{s}$ with leading term $\left[Q_{I_{1}} \iota_{n}|\ldots| Q_{I_{s}} \iota_{n}\right]$ with $\alpha I_{j}>0$ for each $j$, then the proof of Lemma 16 in the case of elements of homological degree one asserts there are arbitrarily large $i$ such that $Q_{i} Q_{I_{1}} \iota_{n}$ has indecomposable leading term $Q_{I_{1}} Q_{i^{\prime}} \iota_{n}$. For such an $i$, $Q_{i+s-1} x$ has $\left[Q_{I_{1}} Q_{i^{\prime}} \iota_{n}\left|Q_{1} Q_{I_{2}} \iota_{n}\right| \ldots \mid Q_{1} Q_{I_{s}} \iota_{n}\right]$ as its greatest term with no leading zeros. Hence $Q_{i+s-1} x$ is not a boundary and the theorem is proved.

\section{Properties of the Hochschild homology spectral sequence}

We have been as restrictive as possible in our definition of topological Hochschild homology in order to avoid as many technical difficulties as possible. The definition we have given is a specialization of one given in [10].

Proof of Theorem 8 . The spectral sequence is the homotopy spectral sequence for the simplicial $H \mathbb{Z} / p$-module CB. $(A ; B)$. Functoriality is clear from this definition. statements (2) and (3) follow from the fact [5, VII.6.5,VII.6.8] that when $A$ and $B$ are $q$-cofibrant commutative $H \mathbb{Z} / p$-algebras $\mathrm{CB} \bullet(A ; B)$ is proper. Statement (4) follows because $\wedge$ is pushout in $\mathcal{E}$ so term by term multiplication yields a map of

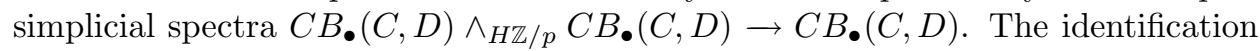
of $E_{* *}^{2}$ in statement (5) also follows from [5, VII.6.8] because $H \mathbb{Z} / p \wedge_{S} \mathbb{C} \Sigma^{\infty} S^{n}$ is in the collection of $H \mathbb{Z} / p$-modules $\overline{\mathcal{E}}_{H \mathbb{Z} / p}$. To deduce the rest of statement (5), let 
$K=\left(H \mathbb{Z} / p \wedge_{S} \mathbb{C} \Sigma^{\infty} S^{n}\right)$ and consider the diagram

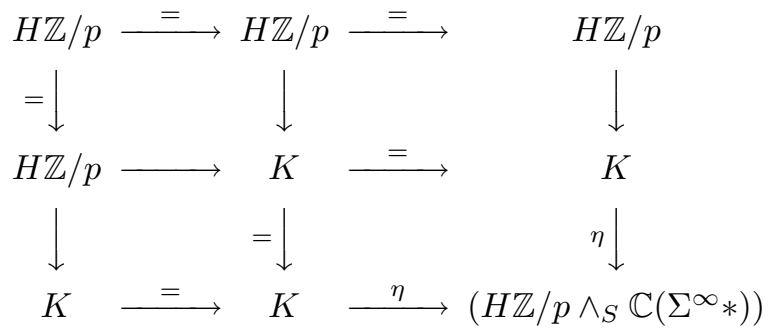

where $\eta$ is induced by the constant map from $S^{n}$ to the point $*$. Applying the cyclic bar construction at each stage yields a sequence

$$
\mathrm{CB} \bullet(H \mathbb{Z} / p ; K) \rightarrow \mathrm{CB} \bullet(K ; K) \rightarrow \mathrm{CB}_{\bullet}\left(K ;\left(H \mathbb{Z} / p \wedge_{S} \mathbb{C}\left(S^{0}\right)\right)\right) .
$$

The usual bar construction spectral sequence mentioned in statement (5) is the homology spectral sequence for the simplicial space $B_{\bullet}\left(Q S^{n}\right)$ which in turn is the homotopy spectral sequence for the simplicial spectrum $H \mathbb{Z} / p \wedge B_{\bullet}\left(Q S^{n}\right)$. (Here $B$. is the usual bar construction.) The weak equivalences $H \mathbb{Z} / p \wedge \Sigma^{\infty} Q S_{+}^{n} \simeq$ $H \mathbb{Z} / p \wedge \mathbb{C} S^{n}$ and $H \mathbb{Z} / p \simeq\left(H \mathbb{Z} / p \wedge_{S} \Sigma^{\infty} *\right)$ thus induce a level-wise weak equivalence between the third term in the sequence (3) and $H \mathbb{Z} / p \wedge B_{\bullet}\left(Q S^{n}\right)$. Hence the spectral sequence for the third term in (3) is the usual bar construction spectral sequence. The first term in (3) is the constant simplicial spectrum $\left(H \mathbb{Z} / p \wedge_{S} \mathbb{C}\left(S^{0}\right)\right)$. The first map admits a left inverse by multiplying all the terms together at each level, again using the fact that $\wedge$ is a pushout. The cogroup structure then yields a map from the center term of (3) to the (termwise) coproduct of the outer terms. This last map yields an isomorphism of spectral sequences since it is an isomorphism of $E^{2}$ terms. (The fact that it is an isomorphism of $E_{2}$ terms follows from applying the argument just given to the cyclic bar construction $\mathrm{CB} \bullet\left(H \mathbb{Z} / p_{*} Q S_{+}^{n} ; H \mathbb{Z} / p_{*} Q S_{+}^{n}\right)$ in the category of graded $\mathbb{Z} / p$ algebras.) Since the middle term in (3) is the Hochschild homology spectral sequence referred to in statement (5) the statement follows.

Remark 19. On $E^{2}$ terms, we get a split short exact sequence of Hopf algebras

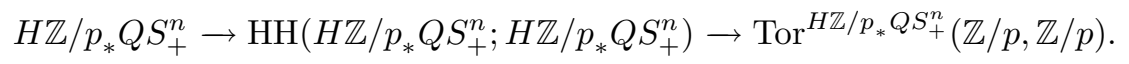

In general if $H$ is a unital augmented commutative graded Hopf algebra there is a split short exact sequence of Hopf algebras

$$
H \rightarrow \mathrm{HH}(H ; H) \rightarrow \operatorname{Tor}^{H}(\mathbb{Z} / p, \mathbb{Z} / p) .
$$

However, the splitting map $\operatorname{Tor}^{H}(\mathbb{Z} / p, \mathbb{Z} / p) \rightarrow \mathrm{HH}(H ; H)$ is not the usual inclusion. For example, if $\psi x=x \otimes 1+y \otimes y+1 \otimes x$ where $x$ and $y$ are polynomial generators, then $\psi[x]=[x] \otimes 1+y \otimes[y]+[y] \otimes y+1 \otimes[x]$. Therefore the usual inclusion does not commute with the coproduct. In our case, $H \mathbb{Z} / p_{*} Q S_{+}^{n}$ is a primitively generated free commutative algebra, so we do get the usual inclusion at $E^{2}$.

\section{REFERENCES}

1. Marcel Bökstedt, The topological Hochschild homology of $\mathbb{Z}$ and $\mathbb{Z} / p$, unpublished.

2. R. R. Bruner, J. P. May, J. E. McClure, and M. Steinberger, $H_{\infty}$ ring spectra and their applications, Lecture Notes in Mathematics, vol. 1176, Springer-Verlag, 1986. MR 88e:55001

3. H. E. A. Campbell, F. P. Peterson, and P. S. Selick, Self-maps of loop spaces I, Transactions of the American Mathematical Society 293 (1986), no. 1, 1-51. MR 87e:55010a 
4. F. R. Cohen, T. J. Lada, and J. P. May, The homology of iterated loop spaces, Lecture Notes in Mathematics, vol. 533, Springer-Verlag, 1976. MR 55:9096

5. A. D. Elmendorf, I. Kriz, M. A. Mandell, and J. P. May, Rings, modules and algebras in stable homotopy theory, Preprint.

6. S. Kochman, Symmetric Massey products and a Hirsch formula in homology, Trans. Amer. Math. Soc. 163 (1972), 245-260. MR 48:9721

7. L. G. Lewis, Jr., J. P. May, and M. Steinberger, Equivariant stable homotopy theory, Lecture Notes in Mathematics, vol. 1213, Springer-Verlag, 1986, with contributions by J. E. McClure. MR 88e: 55002

8. Hans Ligaard and Ib Madsen, Homology operations in the Eilenberg-Moore spectral sequence, Mathematische Zeitschrift 143 (1975), 45-54. MR 51:11511

9. J. P. May, A general algebraic approach to Steenrod operations, The Steenrod Algebra and Its Applications, Lecture Notes in Mathematics, vol. 168, Springer-Verlag, 1970, pp. 153-231. MR 43:6915

10. J. McClure, R. Schwänzl, and $\mathrm{R}$. Vogt, $\mathrm{THH}(R) \cong R \otimes S^{1}$ for $E_{\infty}$ ring spectra, To appear in The Journal of Pure and Applied Algebra.

11. D. C. Ravenel and W. S. Wilson, The Morava K-theories of Eilenberg-MacLane spaces and the Conner-Floyd conjecture, Amer. Jour. Math. 102 (1980), 691-748. MR 81i:55005

Department of Mathematics and Statistics, Swarthmore College, Swarthmore, PennSYLVANIA 19081

E-mail address: thunter1@swarthmore.edu 